\title{
Article \\ Nonlinear Neutral Delay Differential Equations of Fourth-Order: Oscillation of Solutions
}

\author{
Ravi P. Agarwal ${ }^{1,+}$, Omar Bazighifan ${ }^{2,3,+} \mathbb{D}$ and Maria Alessandra Ragusa $4,5, *,+(\mathbb{D}$ \\ 1 Department of Mathematics, Texas A and M University, Kingsville, TX 78363, USA; Ravi.Agarwal@tamuk.edu \\ 2 Department of Mathematics, Faculty of Science, Hadhramout University, Hadhramout 50512, Yemen, \\ o.bazighifan@gmail.com \\ 3 Department of Mathematics, Faculty of Education, Seiyun University, Hadhramout 50512, Yemen \\ 4 Department of Mathematics and Computer Science, University of Catania, 95124 Catania, Italy \\ 5 RUDN University, 6 Miklukho, Maklay St, Moscow 117198, Russia \\ * Correspondence: mariaalessandra.ragusa@unict.it \\ + These authors contributed equally to this work.
}

check for updates

Citation: Agarwal, R.P.; Bazighifan, O.; Ragusa, M.A. Nonlinear Neutral Delay Differential Equations of Fourth-Order: Oscillation of Solutions. Entropy 2021, 23, 129. https://doi.org/10.3390/e23020129

Received: 15 December 2020 Accepted: 15 January 2021 Published: 20 January 2021

Publisher's Note: MDPI stays neutral with regard to jurisdictional clai$\mathrm{ms}$ in published maps and institutional affiliations.

Copyright: $(\odot 2021$ by the authors. Licensee MDPI, Basel, Switzerland. This article is an open access article distributed under the terms and conditions of the Creative Commons Attribution (CC BY) license (https:// creativecommons.org/licenses/by/ $4.0 /)$.

\begin{abstract}
The objective of this paper is to study oscillation of fourth-order neutral differential equation. By using Riccati substitution and comparison technique, new oscillation conditions are obtained which insure that all solutions of the studied equation are oscillatory. Our results complement some known results for neutral differential equations. An illustrative example is included.
\end{abstract}

Keywords: neutral differential equations; oscillation; fourth-order differential equation; $p$-Laplacian equations

\section{Introduction}

In this paper, we study the oscillatory properties of solutions of the following fourthorder neutral differential equation

$$
\left(r(x)\left|w^{\prime \prime \prime}(x)\right|^{p_{1}-2} w^{\prime \prime \prime}(x)\right)^{\prime}+q(x)\left|y\left(g_{1}(x)\right)\right|^{p_{2}-2} y\left(g_{1}(x)\right)=0,
$$

where

$$
w(x)=y(x)+\delta(x) y\left(g_{2}(x)\right)
$$

and subject to the following conditions:

$\left(W_{1}\right) \quad p_{1}, p_{2}>1$ are constants;

$\left(W_{2}\right) \quad r, \delta \in C\left[x_{0}, \infty\right), q \in C\left[x_{0}, \infty\right), q(x)>0, r(x)>0, r^{\prime}(x) \geq 0, p_{2} \geq p_{1}$, $0 \leq \delta(x)<\delta_{0}<\infty, q(x)$ is not identically zero for large $x$,

$\left(W_{3}\right) \quad g_{2} \in C^{1}\left[x_{0}, \infty\right), g_{1} \in C^{1}\left(\left[x_{0}, \infty\right), \mathbb{R}\right), g_{2}^{\prime}(x)>0, g_{1}^{\prime}(x)>0, g_{2}(x) \leq x$, $\lim _{x \rightarrow \infty} g_{2}(x)=\lim _{x \rightarrow \infty} g_{1}(x)=\infty$;

$\left(W_{4}\right) \quad \int_{x_{0}}^{\infty} r^{-1 /\left(p_{1}-1\right)}(s) \mathrm{d} s=\infty$.

Definition 1. A solution of (1) is said to be oscillatory if it has arbitrarily large zeros on $\left[x_{y}, \infty\right)$. Otherwise, a solution that is not oscillatory is said to be nonoscillatory.

Definition 2. The Equation (1) is said to be oscillatory if every solution of it is oscillatory.

Definition 3. A differential equation is said to be neutral if the highest-order derivative of the unknown function appears both with and without delay.

Neutral differential equations are used in numerous applications in technology and natural science. For instance, the problems of oscillatory behavior of neutral differential 
equations have a number of practical applications in the study of distributed networks containing lossless transmission lines which arise in high-speed computers where the lossless transmission lines are used to interconnect switching circuits, see [1-4] . In fact, half-linear differential equations arise in a variety of real world problems such as in the study of $p$-Laplace equations non-Newtonian fluid theory, the turbulent flow of a polytrophic gas in a porous medium, and so forth; see [5-7]. During the past few years there has been interest by many researchers to study the oscillatory behavior of this type of equation, see [8-12]. Furthermore, many researchers investigate regularity and existence properties of solutions to difference equations; see [13-15] and the references therein.

In [16], the authors studied oscillation conditions for equation

$$
\left(r(t) \Phi\left(y^{(n-1)}(t)\right)\right)^{\prime}+a(t) \Phi\left(y^{(n-1)}(t)\right)+q(t) \Phi(y(g(t)))=0,
$$

where $\Phi=|s|^{p-2} s$ and $n$ is even. The authors used Riccati substitution together with integral averaging technique.

In [17], Bazighifan obtained oscillation conditions for solutions of (1) and used comparison method with second-order equations. Moreover, in $[16,18,19]$, the authors considered the equation

$$
\left(r(x)\left|w^{\prime \prime \prime}(x)\right|^{p-2} w^{\prime \prime \prime}(x)\right)^{\prime}+q(x)|y(\delta(x))|^{p-2} y(\delta(x))=0,
$$

where $w(x)=y(x)+\delta(x) y\left(g_{2}(x)\right)$ and obtained a condition under which every solution of this equation is oscillatory.

Bazighifan and Abdeljawad [20] give some results providing information on the asymptotic behavior of solutions of fourth-order advanced differential equations. This time, the authors used comparison method with first and second-order equations.

In this article, we establish oscillatory properties of solutions of (1). By using Riccati substitution and comparison technique, new oscillatory criteria for (1) are established. Our results complement some known results in literature. Furthermore, an illustrative example is provided.

\section{Lemmas}

The following lemmas will be used to establish our main results:

Lemma 1. [21] Let $\beta$ be a ratio of two odd numbers, $D>0$ and $G$ are constants. Then

$$
G y-D y^{(\beta+1) / \beta} \leq \frac{\beta^{\beta}}{(\beta+1)^{\beta+1}} \frac{G^{\beta+1}}{D^{\beta}} .
$$

Lemma 2. [22] Let $y \in C^{n}\left(\left[x_{0}, \infty\right),(0, \infty)\right)$. Assume that $y^{(n)}(x)$ is of fixed sign and not identically zero on $\left[x_{0}, \infty\right)$ and that there exists a $x_{1} \geq x_{0}$ such that $y^{(n-1)}(x) y^{(n)}(x) \leq 0$ for all $x \geq x_{1}$. If $\lim _{x \rightarrow \infty} y(x) \neq 0$, then for every $\mu \in(0,1)$ there exists $x_{\mu} \geq x_{1}$ such that

$$
y(x) \geq \frac{\mu}{(n-1) !} x^{n-1}\left|y^{(n-1)}(x)\right| \text { for } x \geq x_{\mu}
$$

Lemma 3. [23] Let $y^{(i)}(x)>0, i=0,1, . ., n$, and $y^{(n+1)}(x)<0$. Then

$$
\frac{y(x)}{x^{n} / n !} \geq \frac{y^{\prime}(x)}{x^{n-1} /(n-1) !} .
$$

Lemma 4. [3] Let $c, v \geq 0$ and $\beta$ be a positive real number. Then

$$
(c+v)^{\beta} \leq 2^{\beta-1}\left(c^{\beta}+v^{\beta}\right), \text { for } \beta \geq 1
$$


and

$$
(c+v)^{\beta} \leq c^{\beta}+v^{\beta}, \text { for } \beta \leq 1 .
$$

We consider the following notations:

$$
\begin{gathered}
\delta_{1}(x)=\frac{1}{\delta\left(g_{2}^{-1}(x)\right)}\left(1-\frac{\left(g_{2}^{-1}\left(g_{2}^{-1}(x)\right)\right)^{3}}{\left(g_{2}^{-1}(x)\right)^{3} \delta\left(g_{2}^{-1}\left(g_{2}^{-1}(x)\right)\right)}\right) \\
\tilde{R}(x)=\left(\frac{\mu\left(g_{2}^{-1}(\varphi(x))\right)^{3}}{6}\right)^{\left(p_{2}-1\right)} q(x) \delta_{1}^{p_{2}-1}(\varphi(x)) r^{-\left(p_{2}-1\right) /\left(p_{1}-1\right)}\left(g_{2}^{-1}(\varphi(x))\right), \\
R(x)=\int_{x}^{\infty}\left(\frac{1}{r(\varrho)} \int_{\varrho}^{\infty} q(s)\left(\frac{g_{2}^{-1}(\sigma(s))}{s}\right)^{p_{2}-1} \mathrm{~d} s\right)^{1 /\left(p_{1}-1\right)} \mathrm{d} \varrho
\end{gathered}
$$

and

$$
\delta_{2}(x)=\frac{1}{\delta\left(g_{2}^{-1}(x)\right)}\left(1-\frac{\left(g_{2}^{-1}\left(g_{2}^{-1}(x)\right)\right)}{\left(g_{2}^{-1}(x)\right) \delta\left(g_{2}^{-1}\left(g_{2}^{-1}(x)\right)\right)}\right) .
$$

The following lemma summarizes the situations that are discussed in the proofs of our results.

Lemma 5. [24] Let y be an eventually positive solution of (1). Then there exist two possible cases:

$$
\begin{aligned}
& \left(\mathbf{S}_{1}\right) w(x)>0, w^{\prime}(x)>0, w^{\prime \prime}(x)>0, w^{\prime \prime \prime}(x)>0, w^{(4)}(x)<0 \\
& \left(\mathbf{S}_{2}\right) w(x)>0, w^{\prime}(x)>0, w^{\prime \prime}(x)<0, w^{\prime \prime \prime}(x)>0, w^{(4)}(x)<0,
\end{aligned}
$$

for $x \geq x_{1}$, where $x_{1} \geq x_{0}$ is sufficiently large.

\section{Main Results}

Lemma 6. Let $y$ be an eventually positive solution of (1). Then

$$
y(x) \geq \frac{1}{\delta\left(g_{2}^{-1}(x)\right)}\left(w\left(g_{2}^{-1}(x)\right)-\frac{1}{\delta\left(g_{2}^{-1}\left(g_{2}^{-1}(x)\right)\right)} w\left(g_{2}^{-1}\left(g_{2}^{-1}(x)\right)\right)\right) .
$$

Proof. Let $y$ be an eventually positive solution of (1). From the definition of $w$, we see that

$$
\delta(x) y\left(g_{2}(x)\right)=w(x)-y(x)
$$

and so

$$
\delta\left(g_{2}^{-1}(x)\right) y(x)=w\left(g_{2}^{-1}(x)\right)-y\left(g_{2}^{-1}(x)\right) .
$$

Repeating the same process, we obtain

$$
y(x)=\frac{1}{\delta\left(g_{2}^{-1}(x)\right)}\left(w\left(g_{2}^{-1}(x)\right)-\left(\frac{w\left(g_{2}^{-1}\left(g_{2}^{-1}(x)\right)\right)}{\delta\left(g_{2}^{-1}\left(g_{2}^{-1}(x)\right)\right)}-\frac{y\left(g_{2}^{-1}\left(g_{2}^{-1}(x)\right)\right)}{\delta\left(g_{2}^{-1}\left(g_{2}^{-1}(x)\right)\right)}\right)\right),
$$


which yields

$$
y(x) \geq \frac{w\left(g_{2}^{-1}(x)\right)}{\delta\left(g_{2}^{-1}(x)\right)}-\frac{1}{\delta\left(g_{2}^{-1}(x)\right)} \frac{w\left(g_{2}^{-1}\left(g_{2}^{-1}(x)\right)\right)}{\delta\left(g_{2}^{-1}\left(g_{2}^{-1}(x)\right)\right)} .
$$

Thus, (3) holds.

The first result of the paper is a theorem providing oscillation criterion for Equation (1). For this purpose, we employ the Riccati method.

Theorem 1. Let $_{1}(x) \leq g_{2}(x)$. Assume that there exist positive functions $\theta, \theta_{1} \in C^{1}\left(\left[x_{0}, \infty\right), \mathbb{R}\right)$, $\mu_{1} \in(0,1)$ and for every constants $M_{1}, M_{2}>0$ such that

$$
\int_{x_{0}}^{\infty}\left(\eta_{1}(s)-\frac{2^{p_{1}-1}}{p_{1}^{p_{1}}} \frac{r\left(g_{2}^{-1}\left(g_{1}(s)\right)\right)\left(\theta^{\prime}(s)\right)^{p_{1}}}{\left(\mu_{1} \theta(s)\left(g_{2}^{-1}\left(g_{1}(s)\right)\right)^{\prime}\left(g_{1}(s)\right)^{\prime}\left(g_{2}^{-1}\left(g_{1}(s)\right)\right)^{2}\right)^{p_{1}-1}}\right) \mathrm{d} s=\infty
$$

and

$$
\int_{x_{0}}^{\infty}\left(\eta_{2}(s)-\frac{\left(\theta_{1}^{\prime}(s)\right)^{2}}{4 \theta_{1}(s)}\right) \mathrm{d} s=\infty
$$

where

$$
\eta_{1}(x)=M_{1}^{p_{2}-p_{1}} \theta(x) q(x) \delta_{1}^{p_{2}-1}\left(g_{1}(x)\right)
$$

and

$$
\eta_{2}(x)=\delta_{2}^{\left(p_{2}-1\right) /\left(p_{1}-1\right)} \theta_{1}(x) M_{2}^{\left(p_{2}-p_{1}\right) /\left(p_{1}-1\right)} \int_{x}^{\infty}\left(\frac{1}{r(\varrho)} \int_{\varrho}^{\infty} q(s)\left(\frac{g_{2}^{-1}\left(g_{1}(s)\right)}{s}\right)^{p_{2}-1} \mathrm{~d} s\right)^{1 /\left(p_{1}-1\right)} \mathrm{d} \varrho
$$

then (1) is oscillatory.

Proof. Let $y$ be a non-oscillatory solution of (1) on $\left[x_{0}, \infty\right)$. Without loss of generality, we can assume that $y$ is eventually positive. It follows from Lemma 5 that there exist two possible cases $\left(\mathbf{S}_{1}\right)$ and $\left(\mathbf{S}_{2}\right)$.

Let $\left(\mathbf{S}_{1}\right)$ hold. From Lemma 3, we obtain

$$
\frac{w^{\prime}(x)}{w(x)} \leq \frac{3}{x}
$$

Integrating from $g_{2}^{-1}(x)$ to $x$, we find

$$
\frac{w\left(g_{2}^{-1}(x)\right)}{w(x)} \geq \frac{\left(g_{2}^{-1}(x)\right)^{3}}{x^{3}} .
$$

This yields

$$
\left(g_{2}^{-1}(x)\right)^{3} w\left(g_{2}^{-1}\left(g_{2}^{-1}(x)\right)\right) \leq\left(g_{2}^{-1}\left(g_{2}^{-1}(x)\right)\right)^{3} w\left(g_{2}^{-1}(x)\right) .
$$


From (3) and (7), we get

$$
\begin{aligned}
y(x) & \geq \frac{w\left(g_{2}^{-1}(x)\right)}{\delta\left(g_{2}^{-1}(x)\right)}\left(1-\frac{\left(g_{2}^{-1}\left(g_{2}^{-1}(x)\right)\right)^{3}}{\left(g_{2}^{-1}(x)\right)^{3} \delta\left(g_{2}^{-1}\left(g_{2}^{-1}(x)\right)\right)}\right) \\
& \geq \delta_{1}(x) w\left(g_{2}^{-1}(x)\right) .
\end{aligned}
$$

From (1) and (8), we obtain

$$
\left(r(x)\left(w^{\prime \prime \prime}(x)\right)^{p_{1}-1}\right)^{\prime}+q(x) \delta_{1}^{p_{2}-1}\left(g_{1}(x)\right) w^{p_{2}-1}\left(g_{2}^{-1}\left(g_{1}(x)\right)\right) \leq 0 .
$$

Define

$$
\psi_{1}(x):=\theta(x) \frac{r(x)\left(w^{\prime \prime \prime}(x)\right)^{p_{1}-1}}{w^{p_{1}-1}\left(g_{2}^{-1}\left(g_{1}(x)\right)\right)} .
$$

From (9) and (10), we obtain

$$
\begin{aligned}
\psi_{1}^{\prime}(x) \leq & \frac{\theta^{\prime}(x)}{\theta(x)} \psi_{1}(x)-\theta(x) w^{p_{2}-p_{1}}\left(g_{2}^{-1}\left(g_{1}(x)\right)\right) q(x) \delta_{1}^{p_{2}-1}\left(g_{1}(x)\right) \\
& -\left(p_{1}-1\right) \theta(x) \frac{r(x)\left(w^{\prime \prime \prime}(x)\right)^{p_{1}-1}\left(g_{2}^{-1}\left(g_{1}(x)\right)\right)^{\prime}\left(g_{1}(x)\right)^{\prime} w^{\prime}\left(g_{2}^{-1}\left(g_{1}(x)\right)\right)}{w^{p_{1}}\left(g_{2}^{-1}\left(g_{1}(x)\right)\right)} .
\end{aligned}
$$

Recalling that $r(x)\left(w^{\prime \prime \prime}(x)\right)^{p_{1}-1}$ is decreasing, we find

$$
r\left(g_{2}^{-1}\left(g_{1}(x)\right)\right)\left(w^{\prime \prime \prime}\left(g_{2}^{-1}\left(g_{1}(x)\right)\right)\right)^{p_{1}-1} \geq r(x)\left(w^{\prime \prime \prime}(x)\right)^{p_{1}-1} .
$$

This yields

$$
\left(w^{\prime \prime \prime}\left(g_{2}^{-1}\left(g_{1}(x)\right)\right)\right)^{p_{1}-1} \geq \frac{r(x)}{r\left(g_{2}^{-1}\left(g_{1}(x)\right)\right)}\left(w^{\prime \prime \prime}(x)\right)^{p_{1}-1} .
$$

It follows from Lemma 2 that

$$
w^{\prime}\left(g_{2}^{-1}\left(g_{1}(x)\right)\right) \geq \frac{\mu_{1}}{2}\left(g_{2}^{-1}\left(g_{1}(x)\right)\right)^{2} w^{\prime \prime \prime}\left(g_{2}^{-1}\left(g_{1}(x)\right)\right),
$$

for all $\mu_{1} \in(0,1)$. Thus, by (11)-(13), we get

$$
\begin{aligned}
\psi_{1}^{\prime}(x) \leq & \frac{\theta^{\prime}(x)}{\theta(x)} \psi_{1}(x)-\theta(x) w^{p_{2}-p_{1}}\left(g_{2}^{-1}\left(g_{1}(x)\right)\right) q(x) \delta_{1}^{p_{2}-1}\left(g_{1}(x)\right) \\
& -\left(p_{1}-1\right) \theta(x) \frac{\mu_{1}}{2}\left(\frac{r(x)}{r\left(g_{2}^{-1}\left(g_{1}(x)\right)\right)}\right)^{1 /\left(p_{1}-1\right)} \frac{r(x)\left(w^{\prime \prime \prime}(x)\right)^{p_{1}}\left(g_{2}^{-1}\left(g_{1}(x)\right)\right)^{\prime}\left(g_{1}(x)\right)^{\prime}\left(g_{2}^{-1}\left(g_{1}(x)\right)\right)^{2}}{w^{p_{1}}\left(g_{2}^{-1}\left(g_{1}(x)\right)\right)} .
\end{aligned}
$$

Hence,

$$
\begin{aligned}
\psi_{1}^{\prime}(x) \leq & \frac{\theta^{\prime}(x)}{\theta(x)} \psi_{1}(x)-\theta(x) w^{p_{2}-p_{1}}\left(g_{2}^{-1}\left(g_{1}(x)\right)\right) q(x) \delta_{1}^{p_{2}-1}\left(g_{1}(x)\right) \\
& -\left(p_{1}-1\right) \frac{\mu_{1}}{2}\left(\frac{r(x)}{r\left(g_{2}^{-1}\left(g_{1}(x)\right)\right)}\right)^{1 /\left(p_{1}-1\right)} \frac{\left(g_{2}^{-1}\left(g_{1}(x)\right)\right)^{\prime}\left(g_{1}(x)\right)^{\prime}\left(g_{2}^{-1}\left(g_{1}(x)\right)\right)^{2}}{(r \theta)^{1 /\left(p_{1}-1\right)}(x)} \psi_{1}^{\frac{p_{1}}{\left(p_{1}-1\right)}}(x) .
\end{aligned}
$$


Since $w^{\prime}(x)>0$, there exist $x_{2} \geq x_{1}$ and a constant $M>0$ such that

$$
w(x)>M,
$$

for all $x \geq x_{2}$. Using Lemma 1 , we put

$$
\begin{aligned}
& G=\frac{\theta^{\prime}(x)}{\theta(x)}, D=\left(p_{1}-1\right) \frac{\mu_{1}}{2}\left(\frac{r(x)}{r\left(g_{2}^{-1}\left(g_{1}(x)\right)\right)}\right)^{1 /\left(p_{1}-1\right)} \frac{\left(g_{2}^{-1}\left(g_{1}(x)\right)\right)^{\prime}\left(g_{1}(x)\right)^{\prime}\left(g_{2}^{-1}\left(g_{1}(x)\right)\right)^{2}}{(r \theta)^{1 /\left(p_{1}-1\right)}(x)} \\
& \text { and } y=\psi_{1} \text {, we get } \\
& \qquad \psi_{1}^{\prime}(x) \leq-\eta_{1}(x)+\frac{2^{p_{1}-1}}{p_{1}^{p_{1}}} \frac{r\left(g_{2}^{-1}\left(g_{1}(x)\right)\right)\left(\theta^{\prime}(x)\right)^{p_{1}}}{\left(\mu_{1} \theta(x)\left(g_{2}^{-1}\left(g_{1}(x)\right)\right)^{\prime}\left(g_{1}(x)\right)^{\prime}\left(g_{2}^{-1}\left(g_{1}(x)\right)\right)^{2}\right)^{p_{1}-1}} .
\end{aligned}
$$

This implies that

$$
\int_{x_{1}}^{x}\left(\eta_{1}(s)-\frac{2^{p_{1}-1}}{p_{1}^{p_{1}}} \frac{r\left(g_{2}^{-1}\left(g_{1}(s)\right)\right)\left(\theta^{\prime}(s)\right)^{p_{1}}}{\left(\mu_{1} \theta(s)\left(g_{2}^{-1}\left(g_{1}(s)\right)\right)^{\prime}\left(g_{1}(s)\right)^{\prime}\left(g_{2}^{-1}\left(g_{1}(s)\right)\right)^{2}\right)^{p_{1}-1}}\right) \mathrm{d} s \leq \psi_{1}\left(x_{1}\right),
$$

which contradicts (4).

Let $\left(\mathbf{S}_{2}\right)$ hold. By using Lemma 3, we get

$$
\frac{w^{\prime}(x)}{w(x)} \leq \frac{1}{x}
$$

Integrating from $g_{2}^{-1}(x)$ to $x$, we find

$$
w\left(g_{2}^{-1}\left(g_{1}(x)\right)\right) \geq \frac{g_{2}^{-1}\left(g_{1}(x)\right)}{x} w(x) .
$$

which yields

$$
g_{2}^{-1}(x) w\left(g_{2}^{-1}\left(g_{2}^{-1}(x)\right)\right) \leq g_{2}^{-1}\left(g_{2}^{-1}(x)\right) w\left(g_{2}^{-1}(x)\right) .
$$

From (3) and (16), we have

$$
\begin{aligned}
y(x) & \geq \frac{1}{\delta\left(g_{2}^{-1}(x)\right)}\left(1-\frac{\left(g_{2}^{-1}\left(g_{2}^{-1}(x)\right)\right)}{\left(g_{2}^{-1}(x)\right) \delta\left(g_{2}^{-1}\left(g_{2}^{-1}(x)\right)\right)}\right) w\left(g_{2}^{-1}(x)\right) \\
& =\delta_{2}(x) w\left(g_{2}^{-1}(x)\right),
\end{aligned}
$$

which with (1) gives

$$
\left(r(x)\left(w^{\prime \prime \prime}(x)\right)^{p_{1}-1}\right)^{\prime} \leq-q(x) \delta_{2}^{p_{2}-1}\left(g_{1}(x)\right) w^{p_{2}-1}\left(g_{2}^{-1}\left(g_{1}(x)\right)\right) .
$$

Integrating (17) from $x$ to $\varrho$, we obtain

$$
r(\varrho)\left(w^{\prime \prime \prime}(\varrho)\right)^{p_{1}-1}-r(x)\left(w^{\prime \prime \prime}(x)\right)^{p_{1}-1} \leq-\int_{x}^{\varrho}\left(q(s) \delta_{2}^{p_{2}-1}\left(g_{1}(s)\right) w^{p_{2}-1}\left(g_{2}^{-1}\left(g_{1}(s)\right)\right)\right) \mathrm{d} s .
$$


Letting $\varrho \rightarrow \infty$ in (18) and using (15), we obtain

$$
r(x)\left(w^{\prime \prime \prime}(x)\right)^{p_{1}-1} \geq \delta_{2}^{p_{2}-1}\left(g_{1}(x)\right) w^{p_{2}-1}(x) \int_{x}^{\infty} q(s)\left(\frac{g_{2}^{-1}\left(g_{1}(s)\right)}{s}\right)^{p_{2}-1} \mathrm{~d} s .
$$

Integrating this inequality again from $x$ to $\infty$, we get

$$
w^{\prime \prime}(x) \leq-\delta_{2}^{\left(p_{2}-1\right) /\left(p_{1}-1\right)} w^{\left(p_{2}-1\right) /\left(p_{1}-1\right)}(x) \int_{x}^{\infty}\left(\frac{1}{r(\varrho)} \int_{\varrho}^{\infty} q(s)\left(\frac{g_{2}^{-1}\left(g_{1}(s)\right)}{s}\right)^{p_{2}-1} \mathrm{~d} s\right)^{1 /\left(p_{1}-1\right)} \mathrm{d} \rho,
$$

Now, we define

$$
\psi_{2}(x):=\theta_{1}(x) \frac{w^{\prime}(x)}{w(x)} .
$$

Then $\psi_{2}(x)>0$ for $x \geq x_{1}$. By differentiating $\psi_{2}$ and using (19), we find

$$
\begin{aligned}
\psi_{2}^{\prime}(x)= & \frac{\theta_{1}^{\prime}(x)}{\theta_{1}(x)} \psi_{2}(x)+\theta_{1}(x) \frac{w^{\prime \prime}(x)}{w(x)}-\theta_{1}(x)\left(\frac{w^{\prime}(x)}{w(x)}\right)^{2} \\
\leq & \frac{\theta_{1}^{\prime}(x)}{\theta_{1}(x)} \psi_{2}(x)-\frac{1}{\theta_{1}(x)} \psi_{2}^{2}(x) \\
& -\delta_{2}^{\left(p_{2}-1\right) /\left(p_{1}-1\right)} \theta_{1}(x) w^{\left(p_{2}-p_{1}\right) /\left(p_{1}-1\right)}(x) \int_{x}^{\infty}\left(\frac{1}{r(\varrho)} \int_{\varrho}^{\infty} q(s)\left(\frac{g_{2}^{-1}\left(g_{1}(s)\right)}{s}\right)^{p_{2}-1} \mathrm{~d} s\right)^{1 /\left(p_{1}-1\right)} \mathrm{d} \varrho .
\end{aligned}
$$

Thus, we obtain

$$
\psi_{2}^{\prime}(x) \leq-\eta_{2}(x)+\frac{\theta_{1}^{\prime}(x)}{\theta_{1}(x)} \psi_{2}(x)-\frac{1}{\theta_{1}(x)} \psi_{2}^{2}(x)
$$

Using Lemma 1, we put

$$
G=\frac{\theta_{1}^{\prime}(x)}{\theta_{1}(x)}, D=\frac{1}{\theta_{1}(x)}
$$

and $y=\psi_{2}$, we find

$$
\psi_{2}^{\prime}(x) \leq-\eta_{2}(x)+\frac{\left(\theta_{1}^{\prime}(x)\right)^{2}}{4 \theta_{1}(x)}
$$

Then, we get

$$
\int_{x_{1}}^{x}\left(\eta_{2}(s)-\frac{\left(\theta^{\prime}(s)\right)^{2}}{4 \theta(s)}\right) \mathrm{d} s \leq \psi_{2}\left(x_{1}\right),
$$

which contradicts (5). This completes the proof.

The second result of the paper is a theorem providing oscillation criterion for Equation (1). For this purpose, we employ the comparison method with first-order differential equations.

Theorem 2. Let

$$
\frac{\left(g_{2}^{-1}\left(g_{2}^{-1}(x)\right)\right)^{3}}{\left(g_{2}^{-1}(x)\right)^{3} \delta\left(g_{2}^{-1}\left(g_{2}^{-1}(x)\right)\right)} \leq 1
$$

Assume that there exist positive functions $\varphi, \sigma \in \delta^{1}\left(\left[x_{0}, \infty\right), \mathbb{R}\right)$ satisfying $\varphi(x) \leq g_{1}(x), \varphi(x)<g_{2}(x), \sigma(x) \leq g_{1}(x), \sigma(x)<g_{2}(x), \sigma^{\prime}(x) \geq 0$ and $\lim _{x \rightarrow \infty} \varphi(x)=\lim _{x \rightarrow \infty} \sigma(x)=\infty$. 
If there exists $\varepsilon_{1}, \mu \in(0,1)$ such that the differential equations

$$
u_{1}^{\prime}(x)+\tilde{R}(x) u_{1}^{\left(p_{2}-1\right) /\left(p_{1}-1\right)}\left(g_{2}^{-1}(\varphi(x, a))\right)=0
$$

and

$$
u_{2}^{\prime}(x)+\delta_{2}^{\left(p_{2}-1\right) /\left(p_{1}-1\right)} \varepsilon_{1}\left(g_{2}^{-1}(\sigma(x))\right)^{\left(p_{2}-1\right) /\left(p_{1}-1\right)} R(x) u_{2}^{\left(p_{2}-1\right) /\left(p_{1}-1\right)}\left(g_{2}^{-1}(\sigma(x))\right)=0
$$

are oscillatory, then (1) is oscillatory.

Proof. Proceeding as in the proof of Theorem 1. Let Case $\left(\mathbf{S}_{1}\right)$ hold. Since $\varphi(x) \leq g_{1}(x)$ and $w^{\prime}(x)>0$, we obtain

$$
\left(r(x)\left(w^{\prime \prime \prime}(x)\right)^{p_{1}-1}\right)^{\prime} \leq-q(x) \delta_{1}^{p_{2}-1}(\varphi(x)) w^{p_{2}-1}\left(g_{2}^{-1}(\varphi(x))\right) .
$$

Now, by using Lemma 2, we have

$$
w(x) \geq \frac{\mu}{6} x^{3} w^{\prime \prime \prime}(x),
$$

for some $\mu \in(0,1)$. It follows from (24) and (25) that, for all $\mu \in(0,1)$,

$$
\begin{aligned}
& \left(r(x)\left(w^{\prime \prime \prime}(x)\right)^{p_{1}-1}\right)^{\prime}+\left(\frac{\mu\left(g_{2}^{-1}(\varphi(x))\right)^{3}}{6}\right)^{p_{2}-1} q(x) \delta_{1}^{p_{2}-1}(\varphi(x))\left(w^{\prime \prime \prime}\left(g_{2}^{-1}(\varphi(x))\right)\right)^{p_{2}-1} \leq 0 . \\
& u_{1}(x)=r(x)\left(w^{\prime \prime \prime}(x)\right)^{p_{1}-1} . \\
& u_{1}^{\prime}(x)+\tilde{R}(x) u_{1}^{\left(p_{2}-1\right) /\left(p_{1}-1\right)}\left(g_{2}^{-1}(\varphi(x))\right) \leq 0 . \\
& w^{\prime \prime}(x) \leq-\delta_{2}^{\left(p_{2}-1\right) /\left(p_{1}-1\right)} w^{\left(p_{2}-1\right) /\left(p_{1}-1\right)}\left(g_{2}^{-1}(\sigma(x))\right) \int_{x}^{\infty}\left(\frac{1}{r(\varrho)} \int_{\varrho}^{\infty} q(s)\left(\frac{g_{2}^{-1}(\sigma(s))}{s}\right)^{p_{2}-1} \mathrm{~d} s\right)^{1 /\left(p_{1}-1\right)} \mathrm{d} \varrho .
\end{aligned}
$$

Using Lemma 3, we get that

$$
w(x) \geq \varepsilon_{1} x w^{\prime}(x) .
$$

From (16) and (27), we obtain

$$
w^{\prime \prime}(x) \leq-\delta_{2}^{\left(p_{2}-1\right) /\left(p_{1}-1\right)} \varepsilon_{1}\left(w^{\prime}\left(g_{2}^{-1}(\sigma(x))\right)\right)^{\left(p_{2}-1\right) /\left(p_{1}-1\right)}\left(g_{2}^{-1}(\sigma(x))\right)^{\left(p_{2}-1\right) /\left(p_{1}-1\right)} R(x) .
$$

Now, we choose $u_{2}(x):=w^{\prime}(x)$, thus, we find that $u_{2}$ is a positive solution of

$$
u_{2}^{\prime}(x)+\delta_{2}^{\left(p_{2}-1\right) /\left(p_{1}-1\right)} \varepsilon_{1}\left(g_{2}^{-1}(\sigma(x))\right)^{\left(p_{2}-1\right) /\left(p_{1}-1\right)} R(x) u_{2}^{\left(p_{2}-1\right) /\left(p_{1}-1\right)}\left(g_{2}^{-1}(\sigma(x))\right) \leq 0 .
$$

Using (see [25], [Theorem 1]), we also see that (23) has a positive solution, a contradiction. The proof is complete. 
Example 1. Consider the equation

$$
\left\{\begin{array}{l}
\left(y(x)+16 y\left(\frac{1}{2} x\right)\right)^{(4)}+\frac{q_{0}}{x^{4}} y\left(\frac{1}{3} x\right)=0, \\
t \geq 1, q_{0}>0 .
\end{array}\right.
$$

Let $r(x)=1, p_{1}=p_{2}=2, \delta(x)=16, g_{2}(x)=\frac{1}{2} x, g_{2}^{-1}(x)=2 x, g_{1}(x)=\frac{1}{3} x$ and $q(t)=q_{0} / x^{4}$.

Thus, it is easy to see that

$$
\begin{aligned}
\delta_{1}(x) & =\frac{1}{\delta\left(g_{2}^{-1}(x)\right)}\left(1-\frac{\left(g_{2}^{-1}\left(g_{2}^{-1}(x)\right)\right)^{3}}{\left(g_{2}^{-1}(x)\right)^{3} \delta\left(g_{2}^{-1}\left(g_{2}^{-1}(x)\right)\right)}\right) \\
& =\frac{1}{16}\left(1-\frac{1}{2}\right)=\frac{1}{32} \\
\delta_{2}(x) & =\frac{1}{\delta\left(g_{2}^{-1}(x)\right)}\left(1-\frac{\left(g_{2}^{-1}\left(g_{2}^{-1}(x)\right)\right)}{\left(g_{2}^{-1}(x)\right) \delta\left(g_{2}^{-1}\left(g_{2}^{-1}(x)\right)\right)}\right) \\
& =\frac{1}{16}\left(1-\frac{1}{8}\right)=\frac{7}{128}
\end{aligned}
$$

and

$$
\eta_{1}(x)=\frac{q_{0}}{32 x}, \eta_{2}(x)=\frac{7 q_{0}}{1152 x} .
$$

By applying conditions (4) and (5), we find

$$
\begin{aligned}
& \int_{x_{0}}^{\infty}\left(\eta_{1}(s)-\frac{2^{p_{1}-1}}{p_{1}^{p_{1}}} \frac{r\left(g_{2}^{-1}\left(g_{1}(s)\right)\right)\left(\theta^{\prime}(s)\right)^{p_{1}}}{\left(\mu_{1} \theta(s)\left(g_{2}^{-1}\left(g_{1}(s)\right)\right)^{\prime}\left(g_{1}(s)\right)^{\prime}\left(g_{2}^{-1}\left(g_{1}(s)\right)\right)^{2}\right)^{p_{1}-1}}\right) \mathrm{d} s \\
= & \int_{x_{0}}^{\infty}\left(\frac{q_{0}}{32 s}-\frac{729}{32 s}\right) \mathrm{d} s=\left(\frac{q_{0}}{32}-\frac{729}{32}\right) \int_{x_{0}}^{\infty} \frac{\mathrm{d} s}{s} \\
= & +\infty, \quad \text { if } q_{0}>729
\end{aligned}
$$

and for $\theta_{1}(x)=x$, we get

$$
\begin{aligned}
\int_{x_{0}}^{\infty}\left(\eta_{2}(s)-\frac{\left(\theta_{1}^{\prime}(s)\right)^{2}}{4 \theta_{1}(s)}\right) \mathrm{d} s & =\int_{x_{0}}^{\infty}\left(\frac{7 q_{0}}{1152 s}-\frac{1}{4 s}\right) \mathrm{d} s \\
& =+\infty, \quad \text { if } q_{0}>41.14
\end{aligned}
$$

Hence, from Theorem 1, we conclude that (29) is oscillatory if $q_{0}>729$.

\section{Conclusions}

In this work, by using the comparison and Riccati methods we establish a new oscillation conditions of (1). This new conditions complement some known results for neutral differential equations. Furthermore, in future work we will study the oscillatory behavior of this equation by comparison method with second-order differential equations.

Author Contributions: Conceptualization, R.P.A., O.B. and M.A.R. These authors contributed equally to this work. All authors have read and agreed to the published version of the manuscript.

Funding: This research was funded by RUDN University grant number "Program 5-100". The APC was funded by "RUDN University Program 5-100". 
Institutional Review Board Statement: Not applicable.

Informed Consent Statement: Not applicable.

Data Availability Statement: Not applicable.

Acknowledgments: The last author wish to thank the Community University Research Engagement Centre (CUrE) and the support of "RUDN University Program 5-100".

Conflicts of Interest: The authors declare no conflict of interest.

\section{References}

1. Hale, J.K. Theory of Functional Differential Equations; Springer: New York, NY, USA, 1977.

2. Chatzarakis, G.E.; Elabbasy, E.M.; Bazighifan, O. An oscillation criterion in 4th-order neutral differential equations with a continuously distributed delay. Adv. Differ. Equ. 2019, 336, 1-9.

3. Baculikova, B.; Dzurina, J. Oscillation theorems for second-order nonlinear neutral differential equations. Comput. Math. Appl. 2011, 62, 4472-4478. [CrossRef]

4. Moaaz, O.; Elabbasy, E.M.; Muhib, A. Oscillation criteria for even-order neutral differential equations with distributed deviating arguments. Adv. Differ. Equ. 2019, 2019, 297. [CrossRef]

5. Bazighifan, O.; Elabbasy, E.M.; Moaaz, O. Oscillation of higher-order differential equations with distributed delay. J. Inequal. Appl. 2019, 55, 1-9. [CrossRef]

6. Bazighifan, O.; Ramos, H. On the asymptotic and oscillatory behavior of the solutions of a class of higher-order differential equations with middle term. Appl. Math. Lett. 2020, 107, 106431. [CrossRef]

7. Bartusek, M.; Dosla, Z.; Marini, M. Oscillation for higher order differential equations with a middle term. Bound. Value Probl. 2014, 2014, 48. [CrossRef]

8. Li, T.; Baculikova, B.; Dzurina, J. Oscillatory behavior of second-order nonlinear neutral differential equations with distributed deviating arguments. Bound. Value Probl. 2014, 2014, 68. [CrossRef]

9. Grace, S.; Agarwal, R.; Graef, J. Oscillation theorems for fourth order functional differential equations. J. Appl. Math. Comput. 2009, 30, 75-88. [CrossRef]

10. Gyori, I.; Ladas, G. Oscillation Theory of Delay Differential Equations with Applications; Clarendon Press: Oxford, UK, 1991.

11. Moaaz, O.; Dassios, I.; Bazighifan, O.; Muhib, A. Oscillation Theorems for Nonlinear Differential Equations of Fourth-Order. Mathematics 2020, 8, 520. [CrossRef]

12. Ragusa, M.A. Homogeneous herz spaces and regularity results. Nonlinear Anal. Theory Methods Appl. 2009, 71, e1909-e1914.

13. Grace, S.R.; Alzabut, J. Oscillation results for nonlinear second order difference equations with mixed neutral terms. Adv. Differ. Equ. 2020, 2020, 8. [CrossRef]

14. Alzabut, J.; Bohner, M.; Grace, S.R. Oscillation of nonlinear third-order difference equations with mixed neutral terms. Adv. Differ. Equ. 2021, 2021, 3. [CrossRef]

15. Alzabut, J. On the oscillation of higher-order half-linear delay difference equations. Appl. Math. Inf. Sci. 2012, 6, $423-427$.

16. Liu, S.; Zhang, Q.; Yu, Y. Oscillation of even-order half-linear functional differential equations with damping. Comput. Math. Appl. 2011, 61, 2191-2196. [CrossRef]

17. Bazighifan, O. On the oscillation of certain fourth-order differential equations with p-Laplacian like operator. Appl. Math. Comput. 2020, 386, 125475. [CrossRef]

18. Li, T.; Baculikova, B.; Dzurina, J.; Zhang, C. Oscillation of fourth order neutral differential equations with $p$-Laplacian like operators. Bound. Value Probl. 2014, 56, 41-58. [CrossRef]

19. Zhang, C.; Agarwal, R.; Li, T. Oscillation and asymptotic behavior of higher-order delay differential equations with $p$-Laplacian like operators. J. Math. Anal. Appl. 2014, 409, 1093-1106. [CrossRef]

20. Bazighifan, O.; Abdeljawad, T. Improved Approach for Studying Oscillatory Properties of Fourth-Order Advanced Differential Equations with p-Laplacian Like Operator. Mathematics 2020, 8, 656. [CrossRef]

21. Bazighifan, O.; Kumam, P. Oscillation Theorems for Advanced Differential Equations with p-Laplacian Like Operators. Mathematics 2020, 8, 821. [CrossRef]

22. Agarwal, R.; Grace, S.; O'Regan, D. Oscillation Theory for Difference and Functional Differential Equations; Kluwer Academic Publishers: Dordrecht, The Netherlands, 2000.

23. Kiguradze, I.T.; Chanturiya, T.A. Asymptotic Properties of Solutions of Nonautonomous Ordinary Differential Equations; Kluwer Academic Publishers: Dordrecht, The Netherlands, 1993.

24. Bazighifan, O.; Ruggieri, M.; Scapellato, A. An Improved Criterion for the Oscillation of Fourth-Order Differential Equations. Mathematics 2020, 8, 610. [CrossRef]

25. Philos, C.G. On the existence of non-oscillatory solutions tending to zero at $\infty$ for differential equations with positive delays. Arch. Math. 1981, 36, 168-178. [CrossRef] 